\title{
RAPID REAPPEARANCE OF PLASMODIUM FALCIPARUM AFTER DRUG TREATMENT AMONG SENEGALESE ADULTS EXPOSED TO MODERATE SEASONAL TRANSMISSION
}

\author{
CHEIKH-SADIBOU SOKHNA, FARBA B. K. FAYE, ANDRÉ SPIEGEL, HAMADY DIENG, AND JEAN-FRANÇOIS TRAPE \\ Laboratoire de Paludologie, Institut de Recherche pour le Développement (IRD, formerly ORSTOM), Dakar, Senegal; \\ Unité d'Epidémiologie, Institut Pasteur, Dakar, Senegal
}

\begin{abstract}
To investigate the relationship between the entomologic inoculation rate (EIR) and time to reappearance of malaria parasites after radical treatment under moderate seasonal transmission conditions, a study was undertaken in a mesoendemic area of Senegal where malaria transmission is concentrated over an annual three-month period and averages 12 infective bites per person per year. A three-day course of quinine was administered to 48 asymptomatic adults between 19 and 66 years of age. Malaria transmission and parasitemia were monitored every week for two months and cases of fever or symptoms were investigated as part of a daily clinical surveillance. The proportion of persons reinfected at Days 28, 35, and 56 was $25 \%, 38 \%$, and $54 \%$, respectively. Adults less than 40 years of age had a shorter time to reinfection. In this age group, the median Plasmodium falciparum reappearance time was 28 days, and it was estimated that only one infected mosquito bite was able to induce a patent infection among half of the subjects. Only 8\% (2 of 26) of the reinfections caused a clinical attack. These data are discussed in the light of previous studies conducted among adults naturally exposed to intense perennial transmission or among naive volunteers receiving artificial challenges. Rapid reinfection occurs at very low EIRs and dramatic differences in actual and cumulated exposure to infected mosquito bites poorly affect the median time to reappearance of malaria parasites in endemic populations.
\end{abstract}

\section{INTRODUCTION}

The acquisition of premunition by persons frequently reinfected by Plasmodium falciparum enables them to reduce the level of parasitemia during reinfections and to decrease the incidence, duration, and potential severity of clinical attacks. This control of reinfections by semi-immune persons may be first exerted at the pre-erythrocytic level, limiting the proportion of parasite populations inoculated by the vectors that will go beyond the hepatic stage. ${ }^{1,2}$ If parasites emerge from the liver, the rapid control of the level and duration of the peaks of parasitemia following the first cycles of erythocytic schizogony in semi-immune persons will reduce the incidence of clinical attacks and enable the spontaneous clearance of symptoms within a few hours. ${ }^{3,4}$

To investigate this control of reinfections, a simple method consists of measuring the time to reappearance of $P$. falciparum in the blood of asymptomatic persons after treatment and analyzing the clinical and parasitologic patterns of the recurrent infections. In a study conducted in a population exposed to intense perennial transmission, we have previously shown that age was a major factor affecting time to reappearance of malaria parasites after treatment and that adults successfully resisted a large number of natural challenges before presenting a new infection. ${ }^{5}$ Here we report the results of a study conducted among semi-immune adults exposed to moderate seasonal transmission. In contrast with our previous study, a high proportion of infected mosquito bites were able to induce a patent infection in this cohort of adults. We suggest that for a wide range of transmission levels in endemic areas, low differences exist in the time to reappearance of malaria parasites after treatment.

SUBJECTS, MATERIALS, AND METHODS

Study area. The Niakhar study area is located in the Sahel, $120 \mathrm{~km}$ southeast of Dakar, Senegal, in the heart of the
Senegalese ground-nut basin. This rural area of 29,000 inhabitants has, for several decades, been a regional observatory for population and health studies ${ }^{6,7}$ Rain is concentrated over a three-month period from July to the beginning of October. Annual rainfall averaged $444 \mathrm{~mm}$ from 1984 through 1996. During 1996, the study year, annual rainfall was $545 \mathrm{~mm}$ and the last rain occurred on October 16 .

Entomologic surveys in this area showed that malaria transmission, almost exclusively by Anopheles arabiensis, was strictly seasonal and concentrated in September and October. A survey carried out in 1995, a year of heavy rainfall $(614 \mathrm{~mm})$, indicated an entomologic inoculation rate (EIR) of 12 and 9 infective bites per person per year, respectively, in Diohine village (the place of the present study) and in another representative village of the Niakhar area. ${ }^{8}$ The parasite rate in children is usually less than $50 \%$ most of the year, but can reach $84 \%$ at the end of the rainy season, with rates of $82 \%, 15 \%$, and $0.2 \%$ for $P$. falciparum, $P$. malariae, and $P$. ovale, respectively. ${ }^{9}$ Malaria mortality is concentrated in children less than 10 years old, with approximately $80 \%$ of the malaria deaths among children $0-4$ years of age. From 1988 to 1991 , the average malaria mortality rate was 4.0 per thousand per year among children between 0 and 9 years of age. The emergence of chloroquine resistance in 1992 was associated with a dramatic increase in malaria mortality, which averaged 8.2 per thousand per year during the period 1992-1995.7 The proportion of RII and RIII responses has increased from $10 \%$ in 1993 to $29 \%$ in $1996 .{ }^{10,11}$

Study subjects and protocol. The study was conducted from October 4, 1996 to November 29, 1996 among a cohort of 48 adults volunteers from Diohine village. Criteria for inclusion in the study were living continuously in the village during the whole surveillance period, being in good health, and not taking malaria chemoprophylaxis, and not having left the village until the end of November. The study protocol was carefully explained to the assembled village population and informed consent was individually obtained from 